\section{Scan objects faster and more efficiently}

The Scantist 3D Dental Scan Spray was developed so that dental laboratories can scan objects such as dental braces even faster and more efficiently.

With Scantist 3D, transparent or reflective parts are covered with a matting layer within a few seconds, so that high contrast values and thus precise measurement results can be achieved. The special feature: Scantist 3D completely evaporates within approximately 30 minutes.

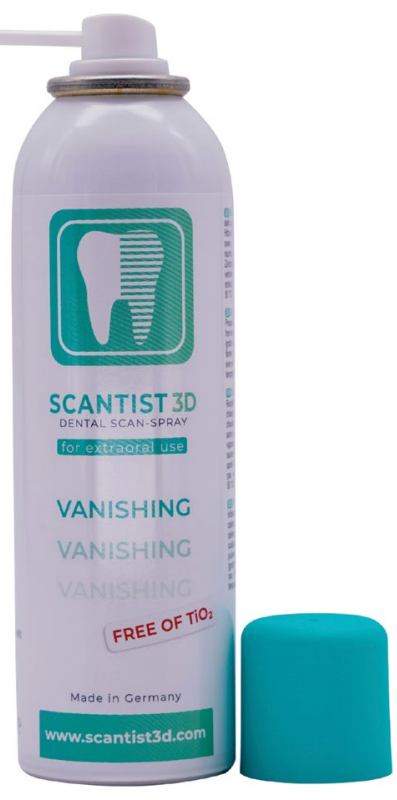

The previously time-consuming and costly removal of the spray layer after the scanning process is no longer necessary. In addition, compared with conventional scanning sprays, Scantist $3 \mathrm{D}$ is pigment-free and therefore gentle on people and the environment.

In addition to transparent and reflective parts, scanning sprays are also used on objects with deep indentations such as telescopes, brackets or mouthpieces. The application of the spray creates a homogeneous and very fine matting layer that prevents reflections and evens out uneven structures on the surface. Improvement of the scanning requirements optimises the CAD/CAM processes and leads to significant cost and time savings.

www.scantist-3d.com

\title{
Protect gums and the planet with $100 \%$ recyclable toothpaste
}

Boasting a $100 \%$ recyclable carton, tube and cap, vegan-friendly formula, and clinically proven to improve gum health, the Arm \& Hammer 100\% Natural Gum Protection Toothpaste is perfect for patients wanting to do good and feel good.

In the brand-new $100 \%$ natural toothpaste range from Arm \& Hammer, the $100 \%$ Natural Gum Protection Toothpaste is powered by baking soda - gently removing plaque and stains to leave patients with a whiter, brighter smile and long-lasting oral health.

Its benefits include:

- Remineralises tooth enamel

- Neutralises acids caused by everyday food and drink

- Returns oral $\mathrm{pH}$ to neutral levels

- Provides lasting freshness

- $100 \%$ recyclable (carton, tube and cap)

- No preservatives

- No artificial colours

- Vegan-friendly

- Quickly dissolves to clean the total tooth surface fast

- Deep cleans by getting between teeth and right up to the gum line

- Clinically proven.

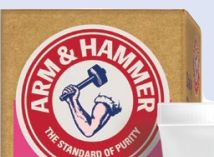

$100 \%$

Natura

Baking

Soda

Goothp

\begin{tabular}{l} 
Gum \\
Protection \\
\hline
\end{tabular}

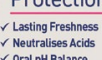

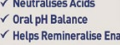

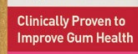

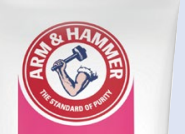

$100 \%$

Natural

Baking

Soda

Gum
Protection

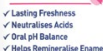

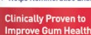

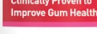
$\mathrm{V}$

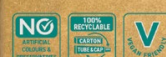

For more information about the carefully formulated Arm \& Hammer toothpaste range, visit https://www. armandhammer.co.uk/ or email: ukenquiries@ churchdwight.com.

Arm \& Hammer 100\% Natural Baking Soda Toothpaste range can now be purchased from Boots, Amazon and Superdrug, with further stockists following.

\section{Elevate your practice}

Dentally has launched Elevate - a new way to support you and your practice at every step of your journey, and as part of its commitment to leveraging the power of cloud-enabled practice management software in your dental practice.

The Elevate programme provides your practice with a consultant $100 \%$ dedicated to seeking to understand your practice priorities and aspirations. Your adviser will tailor the support they provide to your individual needs, supporting you and helping you understand your business opportunities and optimise your workflow efficiencies backed by benchmarked data and delivering you tangible outcomes like so many other practices across the UK.

After the tumultuous few years dental practices across the UK have had to deal with as a result of COVID-19, now more than ever, Dentally's team are there for you to help you find your feet once more and get your practice back up and running in the most efficient and effective of ways. The Elevate programme can help you to do exactly that. Ninety-two percent of Dentally's customers

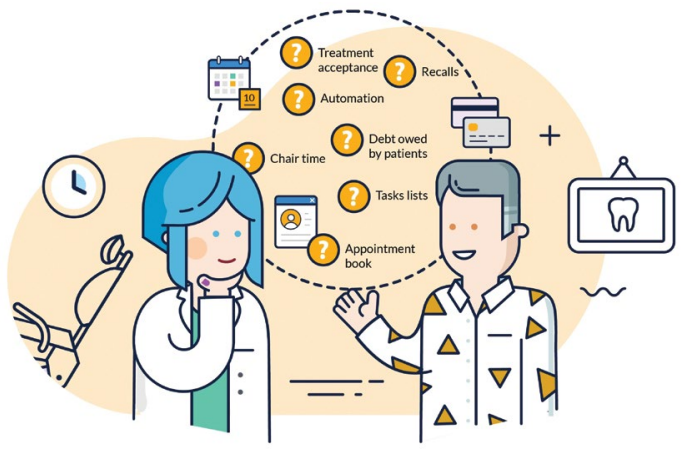

said that the programme had a significant or major impact on their business outcomes in 2021.

For no added cost, just a little bit of dedicated time set aside to talk support and how they can help, the Elevate programme is available to all Dentally customers to help streamline their practices. To book an initial call with your own Elevate consultant, visit the following link and book a convenient time for you online: https://www.dentally. co/uk/about-dentally/elevate. Elevate your practice now and leverage the power of the cloud.

For more information visit: https:// dentally.co/ or call 02038565610 . 